\title{
Phosphate-solubilizing characteristics of yeasts
}

\author{
Kuo CY ${ }^{\dagger}$, Fu SF ${ }^{\dagger}$, Chou FC, Chen RY and Chou JY* \\ Department of Biology, National Changhua University of Education, Changhua 500, Taiwan \\ ${ }^{\dagger}$ These authors contributed equally to this work.
}

Kuo CY, Fu SF, Chou FC, Chen RY, Chou JY 2018 - Phosphate-solubilizing characteristics of yeasts. Mycosphere 9(6), 1117-1131, Doi 10.5943/mycosphere/9/6/4

\begin{abstract}
Phosphorus $(\mathrm{P})$ is an essential element for plant development and metabolism. Because of its low availability and mobility in soils, it is often a limiting nutrient of plant growth. When phosphorus content in soil is insufficient, symptoms of phosphorus deficiency can appear, such as purple spots on leaves and stems or inhibition of development and maturation. To provide adequate nutrients for plant growth, appropriate fertilizers should be applied. However, overuse of chemical fertilizers can cause unanticipated environmental effects. To decrease the negative environmental effects resulting from continued use of chemical fertilizers, we can inoculate plants with phosphatesolubilizing microorganisms. Phosphate-solubilizing microorganisms are crucial in dissolving fertilizer phosphorus and bound phosphorus in soil in a manner that is both environmentally friendly and sustainable. In this study, we selected yeasts with calcium-phosphate-solubilizing ability and found that this ability to be regulated by environmental factors (e.g. amount of soluble phosphate, liquid or solid agar plates condition, and type of inorganic phosphate). Arabidopsis thaliana was inoculated with selected yeasts; we found that Cryptococcus laurentii (JYC370) promoted plant growth in an inorganic phosphate $\left(\mathrm{P}_{\mathrm{i}}\right)$-deficient medium supplemented with calcium phosphate dibasic dihydrate. The amount of cellular inorganic phosphate was also higher in yeasttreated plants than in control plants grown in the $\mathrm{P}_{\mathrm{i}}$-deficient medium. This result reveals the potential of these strains for biofertilizer applications and commercial use as biofertilizer agents in the future.
\end{abstract}

Keywords - Arabidopsis thaliana - biofertilizer - calcium phosphate - phosphorus - phosphatesolubilizing microorganisms

\section{Introduction}

Many elements are required for plant growth. According to the amount required by plants, elements are classified into major and trace elements. Major elements are chemical elements required by plants in large amounts and include nitrogen, phosphorus, and potassium. Trace elements are elements required by plants in small amounts and include iron, manganese, and boron. If plants lack these elements, symptoms of plant diseases can occur, and plant growth can be hindered. Although phosphorus (P) is abundant in soil in both inorganic and organic forms (Gyaneshwar et al. 2002), it is the least mobile and available major nutrient to plants in most soil conditions. Therefore, it is frequently the major or prime limiting factor of plant growth (Hinsinger 2001). Phosphorus is required for seed germination, protein formation, photosynthesis, and 
metabolism, and it also promotes flowering and fruiting (Sultenfuss \& Doyle 1999, Wrage et al. 2010). Depending on the environmental $\mathrm{pH}$, phosphorus exists as several anions in soil $\left(\mathrm{PO}_{4}{ }^{3-}\right.$, $\mathrm{HPO}_{4}{ }^{2-}$, and $\left.\mathrm{H}_{2} \mathrm{PO}_{4}{ }^{-}\right)$; however, plants can only assimilate the inorganic phosphate $\left(\mathrm{P}_{\mathrm{i}}\right)$ forms of $\mathrm{HPO}_{4}{ }^{2-}$ and $\mathrm{H}_{2} \mathrm{PO}_{4}{ }^{-}$, which are present in soil at very low concentrations $0.1-10 \mu \mathrm{M}$ (Hinsinger 2001, Nussaume et al. 2011). Plants have evolved various developmental and biochemical mechanisms to overcome phosphorus deficiency. Developmentally, plants can invest more resources into development and allocate more carbon to the roots (Hermans et al. 2006), resulting in an increased root-shoot biomass ratio (Wrage et al. 2010). Biochemically, the hydrolysis of phospholipids is an important biochemical pathway that can lead to an increase in internal phosphate availability (Cruz-Ramírez et al. 2006). Plants can also improve their internal phosphatase activity or secretion of organic acids and phosphatases, which are required for the mineralization of organic $\mathrm{P}$ to release $\mathrm{P}_{\mathrm{i}}$ into soil (Raghothama 1999, Belgaroui et al. 2016).

To provide adequate nutrients for plant growth, farmers intensively apply $\mathrm{P}$ fertilizers to prevent phosphorus deficiency and thus increase yields (Gilbert 2009). However, overuse of chemical fertilizers can cause unanticipated environmental effects. To reduce the negative environmental effects resulting from the overuse of chemical fertilizers, we can inoculate plants with plant-growth-promoting microorganisms, which benefit plant growth and development (Abhilash et al. 2016, Kumar 2016). Phosphate-solubilizing microorganisms, which are a type of plant-growth-promoting microorganisms, play a crucial role in supplying $\mathrm{P}$ to plants in a manner that is both environmentally friendly and sustainable (Gyaneshwar et al. 2002). Phosphatesolubilizing microorganisms include microorganisms that convert insoluble phosphates into soluble phosphates. These organisms utilize various mechanisms to solubilize phosphates. For example, (1) production of phosphatase: similar to plants, phosphate-solubilizing microorganisms produce phosphatase to catalyze the hydrolysis of phosphate (Ponmurugan \& Gopi 2006). Phosphatases catalyze dephosphorylating reactions that involve the hydrolysis of phosphoester or phosphoanhydride bonds (Rodríguez \& Fraga 1999). Depending on the environmental pH, phosphatases can be acidic or alkaline. Acidic phosphatases (EC 3.1.3.2) are a common class of enzymes that catalyze the hydrolysis of phosphomonoesters at acidic $\mathrm{pH}$ (Behera et al. 2017). Alkaline phosphatases (E.C.3.1.3.1) are hydrolases that function at alkaline $\mathrm{pH}$ (Nalini et al. 2015). (2) Secretion of organic acids: Studies have shown that microorganisms acidify the medium through the production of organic acids or the secretion of $\mathrm{H}^{+}$during phosphate solubilization. Therefore, inorganic phosphate can be released through the substitution of protons for $\mathrm{Ca}^{2+}$ (Mardad et al. 2013, Meena et al. 2017). The secretion of various organic acids by phosphatesolubilizing bacteria is considered the main mechanism of phosphate solubilization (Chen et al. 2006, Patel et al. 2008). The type of acid produced depends on the microorganism. Many organic acids, including citric acid, gluconic acid, lactic acid, and succinic acid, are produced by phosphatesolubilizing bacteria. Among various organic acids, gluconic acid is the main organic acid secreted during solubilization by phosphate-solubilizing bacteria (Goldstein 1995, Vyas \& Gulati 2009). Several researchers have reported that inoculation with phosphate-solubilizing bacteria result in improved growth, yield, and P uptake in several crops (Hameeda et al. 2008, Hu et al. 2010, Yu et al. 2012, Saxena et al. 2013). In addition to bacteria, phosphate-mineralizing fungi, such as Absidia spp. and Penicillium purpurogenum, have been reported to show potential as plant-growthpromoting agents (Gaind \& Nain 2015). The soil fungi belonging to genera Aspergillus and Penicillium have been shown to possess the ability to solubilize sparingly soluble phosphates in vitro by secreting inorganic or organic acids (Whitelaw 1999). However, most studies have investigated the phosphate-solubilizing ability of bacteria and filamentous fungi and have seldom focused on yeasts. The genome of yeasts is more stable than that of bacteria, and they are easier to culture than fungi. Therefore, yeasts may be a favorable candidate for biofertilizers. The aims of this study were as follows: (a) to select yeasts with calcium-phosphate-solubilizing ability, (b) to investigate whether this ability of yeasts is regulated by environmental factors (e.g. amount of soluble phosphate, liquid or solid agar plate condition, and type of inorganic phosphate), and (c) to 
evaluate whether the inoculation of plants with calcium-phosphate-solubilizing yeasts improves their growth.

\section{Materials \& Methods}

\section{Yeast culture condition}

The yeast strains used in this study were isolated from Drosera spatulata leaves in our previous study (Fu et al. 2016). As shown in Table 1, the yeasts selected in this study were strains with the ability to solubilize calcium phosphate dibasic dihydrate $\left(\mathrm{CaHPO}_{4} \cdot 2 \mathrm{H}_{2} \mathrm{O}\right)$ or calcium phosphate tribasic $\left(\mathrm{Ca}_{5}(\mathrm{OH})\left(\mathrm{PO}_{4}\right)_{3}\right)$ on Pikovskaya's agar plate, and some strains without solubilizing ability were selected as the control groups (Amprayn et al. 2012). Before each experiment, yeasts were cultured on Yeast Extract-Peptone-Dextrose (YPD) agar plates (1\% Yeast extract, $2 \%$ Peptone, $2 \%$ Dextrose, and $2 \%(\mathrm{w} / \mathrm{v})$ agar) at $28^{\circ} \mathrm{C}$ in an incubator. The voucher specimens of yeasts used in this study are deposited in the Bioresource Collection and Research Center, Hsinchu City, Taiwan (http://www.bcrc.firdi.org.tw/). Any requests should be addressed to the corresponding author.

Table 1 Yeast strains used in this study.

\begin{tabular}{lllll}
\hline Strains & $\begin{array}{l}\text { calcium } \\
\text { phosphate } \\
\text { dibasic } \\
\text { dehydrate }\end{array}$ & $\begin{array}{l}\text { calcium } \\
\text { phosphate } \\
\text { tribasic }^{\mathbf{1}}\end{array}$ & $\begin{array}{l}\text { MS-calcium } \\
\text { phosphate } \\
\text { dibasic } \\
\text { dehydrate }\end{array}$ & $\begin{array}{l}\text { MS-calcium } \\
\text { phosphate } \\
\text { tribasic }\end{array}$ \\
\hline $\begin{array}{l}\text { Meyerozyma caribbica }(\mathrm{JYC} 358) \\
\text { Candida } \text { sp. (JYC363) }\end{array}$ & No & Yes & Yes & Yes \\
Torulaspora sp. (JYC369) & Yes & Yes & Yes & Yes \\
Cryptococcus laurentii (JYC370) & Yes & Yes & Yes & Yes \\
Pseudozyma sp. (JYC372) & Yes & Yes & Yes & Yes \\
Aureobasidium pullulans $(J Y C 375)$ & Yes & Yes & Yes & Yes \\
Kazachstania jiainicus $(J Y C 361)$ & No & No & No & Yes \\
Hanseniaspora uvarum $(J Y C 364)$ & No & Yes & No & No \\
Hanseniaspora uvarum $(J Y C 365)$ & No & Yes & No & No \\
\hline
\end{tabular}

${ }^{1}$ Data from Fu et al. 2016

\section{Phosphate solubilization measurement}

\section{In vitro screening of yeast for calcium-phosphate-solubilizing activity}

For the phosphate solubilization assay, the yeast strains selected were screened in a $\mathrm{P}_{\mathrm{i}^{-}}$ deficient (-P) quarter-strength Murashige-Skoog (MS) medium ( $\mathrm{pH} 5.7$ ) containing $10 \mathrm{~g}$ of sucrose, $0.5 \mathrm{~g}$ of 2-morpholinoethanesulfonic acid monohydrate, $25 \mathrm{~mL}$ of $10 \times$ macronutrient salts (8.25 g of $\mathrm{NH}_{4} \mathrm{NO}_{3}, 9.5 \mathrm{~g}$ of $\mathrm{KNO}_{3}, 2.2 \mathrm{~g}$ of $\mathrm{CaCl}_{2} \cdot 2 \mathrm{H}_{2} \mathrm{O}, 1.85 \mathrm{~g}$ of $\mathrm{MgSO}_{4} \cdot 7 \mathrm{H}_{2} \mathrm{O}$, and $500 \mathrm{~mL}$ of deionized water), $25 \mathrm{~mL}$ of $10 \times$ minor nutrient salts (M0529, Sigma), $950 \mathrm{~mL}$ of deionized water, solid medium with $1.2 \%$ (w/v) Bacto-agar, and $5 \mathrm{~g}$ of $\mathrm{CaHPO}_{4} \cdot 2 \mathrm{H}_{2} \mathrm{O}$ (04231, Sigma) or $\mathrm{Ca}_{5}(\mathrm{OH})\left(\mathrm{PO}_{4}\right)_{3}(\mathrm{CB} 0263$, Bio Basic Inc.). Yeast suspensions $(3 \mu \mathrm{L})$ were pipetted in the center of (-P) MS agar plates. After 5 (MS-calcium phosphate dibasic dihydrate) or 7 days (MS-calcium phosphate tribasic) of incubation at $28^{\circ} \mathrm{C}$, a visible halo zone around the colonies was observed (caused by the solubilization of calcium phosphate by the yeast). The solubilization efficiency unit of each strain was calculated as the diameter of the entire visible halo zone divided by the diameter of the zone with yeast colonies. The strains that could dissolve calcium phosphate dibasic dihydrate or calcium phosphate tribasic on MS plates were then further tested for quantitative analysis of their calcium phosphate dibasic dihydrate or calcium phosphate tribasic solubilizing ability in liquid medium. 


\section{Quantitative analysis of yeast for phosphate-solubilizing activity}

Quantitative analysis of phosphate-solubilizing yeasts was performed for their calcium phosphate dibasic dehydrate- and calcium phosphate tribasic- solubilizing abilities in liquid MS medium. Yeast strains were inoculated in $12 \mathrm{~mL}$ of MS medium and incubated for 3 days at $28^{\circ} \mathrm{C}$. The yeast cultures were sampled every day to measure the concentration of soluble phosphate and the $\mathrm{pH}$ value. Yeast cultures $(3 \mathrm{~mL})$ were centrifuged at 13,000 rpm for 2 minutes, and the supernatant was transferred to a new microtube. The concentration of inorganic phosphate was measured through the ascorbate method (Ames 1966). The $\mathrm{pH}$ of the supernatant was measured using a pH meter (UltraBasic Benchtop pH Meter, Denver Instrument, Bohemia, NY, USA).

\section{Influence of soluble phosphate on phosphate-solubilizing ability}

Media containing three concentrations of exogenous soluble phosphate $(0,5$, and $20 \mathrm{mM})$ were prepared by using MS agar medium and $100 \mathrm{mM} \mathrm{K} \mathrm{HPO}_{4}$ solution. A yeast suspension (3 $\mu \mathrm{L})$ was inoculated into (-P) MS agar plates containing each of the three soluble phosphate concentrations in triplicate. The SE of each strain was calculated as the diameter of the visible halo zone divided by the diameter of the zone with yeast colonies.

\section{Cocultivation of plants with yeasts}

\section{Plant materials and growth conditions}

All plants used in this study were Arabidopsis thaliana ecotype Columbia (Col-0). The medium used in this experiment was the same as the $\mathrm{P}_{\mathrm{i}}$-deficient medium but with the addition of $0.5 \mathrm{~g}$ of calcium phosphate dibasic dehydrate. Seeds were surface sterilized using $1 \%(\mathrm{v} / \mathrm{v})$ sodium hypochlorite solution with a few drops of Tween 20 for 5 minutes. After washing four times in sterile distilled water, seeds were sown in plates containing the $-\mathrm{P}+$ calcium phosphate dibasic dehydrate medium. The plates were then placed vertically in a plant growth chamber with a photoperiod of 16 -h light and 8-h dark at $22^{\circ} \mathrm{C}$. Yeasts were inoculated at the opposite ends of agar plates containing 14-day-old germinated Arabidopsis seedlings (10 seedlings per plate). The culture condition was as follows: a photoperiod of 16-h light and 8-h dark and $22^{\circ} \mathrm{C}$.

\section{Quantitative analysis of cellular $P_{i}$ in plants}

At 14 days after inoculation, inorganic phosphate was measured. Cellular $\mathrm{P}_{\mathrm{i}}$ content was determined through the ascorbate method (Ames 1966). Briefly, plant tissues were weighed and subsequently submerged in $1 \mathrm{~mL}$ of $1 \%$ glacial acetate. After eight freeze-thaw cycles, $100 \mathrm{~mL}$ of the extract was mixed with $200 \mathrm{~mL}$ of $\mathrm{H}_{2} \mathrm{O}$ and $700 \mathrm{~mL}$ of $\mathrm{P}_{\mathrm{i}}$ reaction buffer $(\mathrm{A}=0.48 \%$ $\mathrm{NH}_{4} \mathrm{MoO}_{4}, 2.86 \%(\mathrm{v} / \mathrm{v}) \mathrm{H}_{2} \mathrm{SO}_{4} ; \mathrm{B}=10 \%(\mathrm{w} / \mathrm{v})$ ascorbic acid; $\left.\mathrm{A}: \mathrm{B}(\mathrm{v} / \mathrm{v})=6: 1\right)$. The reaction was allowed to proceed at $45^{\circ} \mathrm{C}$ for $20 \mathrm{~min}$. $\mathrm{P}_{\mathrm{i}}$ content was determined from the standard curve of $\mathrm{K}_{2} \mathrm{HPO}_{4}$ and is expressed as $\mu \mathrm{mol} \cdot \mathrm{g}^{-1}$ fresh weight (Wang et al. 2012).

\section{Statistical analysis}

Data are expressed as mean \pm standard deviation (SD). The calcium phosphate dibasic dihydrate - or calcium phosphate tribasic-solubilizing ability of different yeast strains and the biomass and cellular $\mathrm{P}_{\mathrm{i}}$ content of Arabidopsis were compared with controls through one-way ANOVA with the least significant difference (when variance was homogeneous) or Games-Howell (when variance was heterogeneous) post hoc test. A value of $P<0.05$ was considered statistically significant.

\section{Results}

\section{Yeasts' in vitro calcium-phosphate-solubilizing activity on solid agar plates}

Phosphorus availability is subject to its chemical fixation in soil with other metal cations, which depends on environmental conditions. In this study, nine strains were used (Table 1); five 
had exhibited in vitro calcium phosphate dibasic dehydrate-solubilizing ability on Pikovskaya's agar plate, and eight had exhibited calcium phosphate tribasic-solubilizing ability on Pikovskaya's agar plate, as reported in our previous study (Fu et al. 2016). The phosphate-solubilizing ability on MS-calcium phosphate dibasic dehydrate and MS-calcium phosphate tribasic plates was different from that on Pikovskaya's agar plate. Interestingly, Meyerozyma caribbica (JYC358) did not show calcium phosphate dibasic dehydrate-solubilizing activity on Pikovskaya's agar plate but showed the activity on MS-calcium phosphate dibasic dehydrate plates. Furthermore, Hanseniaspora uvarum (JYC364 and JYC 365) showed calcium phosphate tribasic-solubilizing activity on Pikovskaya's agar plate but not on MS-calcium phosphate tribasic plates. Among the strains tested, Candida sp. (JYC363) exhibited the strongest solubilizing activity for both calcium phosphate dibasic dehydrate and calcium phosphate tribasic, with solubilization efficiency of 1.72 and 1.9, respectively (Figs. 1, 2, Table 2). Because of the unstable phosphate-solubilizing activity of Pseudozyma sp. (JYC372) on the agar plate, this strain was not further analyzed in the following experiments.

\section{Phosphate-solubilizing ability with different amounts of soluble phosphate}

In this study, for most strains, the diameters of the visible halo zones around the colonies decreased when yeasts were incubated with the highest soluble phosphate concentration used (20 $\mathrm{mM}$ ) (Figs 1,2). This finding suggested that the calcium phosphate dibasic dehydrate- or calcium phosphate tribasic-solubilizing ability of most yeast strains was affected by exposure to a high concentration of soluble phosphate. On the MS-calcium phosphate dibasic dehydrate plates, the solubilizing ability of Torulaspora sp. (JYC369) was induced by low levels of exogenous soluble phosphate $(0 \mathrm{mM})$ and inhibited by high levels of exogenous soluble phosphate (5 and $20 \mathrm{mM})$ (Fig. 1C, Table 2). By contrast, M. caribbica (JYC358), Cryptococcus laurentii (JYC370), and A. pullulans (JYC375) showed the strongest solubilizing ability in the presence of $5 \mathrm{mM}$ soluble phosphate but the weakest ability in the presence of $20 \mathrm{mM}$ soluble phosphate (Fig. 1A, D, F, Table 2). Notably, Candida sp. (JYC363) still dissolved calcium phosphate dibasic dehydrate in the presence of a high concentration of soluble phosphate; Candida sp. (JYC363) may not as influenced by exogenous soluble phosphate as were the other strains (Fig. 1B, Table 2).On MScalcium phosphate tribasic plates, the ratio of the visible halo zone to colony diameter generally showed a decreasing trend as the soluble phosphate concentration increased for all yeast strains used in this study (Fig. 2, Table 3). Both Torulaspora sp. (JYC369) and C. laurentii (JYC370) lost their phosphate-solubilizing ability in the presence of $20 \mathrm{mM}$ soluble phosphate (Fig. 2C, D, Table 3). Among the strains used, the phosphate-solubilizing ability of Candida sp. (JYC363) remained the strongest on MS-calcium phosphate tribasic plates (Fig. 2B, Table 3).

\section{Quantitative analysis of yeasts' phosphate-solubilizing ability in liquid medium}

After screening the calcium-phosphate-solubilizing yeasts on agar plates, we found slight differences in the phosphate-solubilizing ability of the yeast strains between Pikovskaya's agar plate and MS plates with calcium phosphate dibasic dihydrate or calcium phosphate tribasic. Furthermore, the phosphate solubilization efficiency of nine strains were measured through quantitative analysis of the available phosphorus in the MS liquid medium (Fig. 3). In our preliminary test, we cultured yeasts in MS medium with calcium phosphate dibasic dehydrate or calcium phosphate tribasic for 7 days. We found that the solubilizing ability of most strains peaked on the third day and remained steady until day 7 (data not shown). Therefore, the following experiments were conducted in 3 days. In the MS-calcium phosphate dibasic dehydrate liquid medium, Candida sp. (JYC363) exhibited high solubilizing ability $(4.77 \pm 0.09 \mu \mathrm{M})$, whereas $M$. caribbica (JYC358), Torulaspora sp. (JYC369), C. laurentii (JYC370), and A. pullulans (JYC375) showed moderate solubilizing ability $(3.81 \pm 0.84,1.85 \pm 0.34,1.52 \pm 0.34$, and $2.5 \pm 0.42 \mu \mathrm{M}$, respectively). However, Kazachstania jiainicus (JYC361), Hanseniaspora uvarum (JYC364 and JYC 365), and Pseudozyma sp. (JYC372) showed little or no solubilizing ability $(0.30 \pm 0.11,0.35$ $\pm 0.15,0.34 \pm 0.07$, and $-0.29 \pm 0.04 \mu \mathrm{M}$, respectively). The negative value indicates that the 
phosphate dissolved by the yeast was less than that in the medium without yeast inoculation, because the phosphate dissolved was entirely consumed by the yeast. The yeast growth condition of Pseudozyma sp. (JYC372) was similar to other yeasts; therefore, Pseudozyma sp. (JYC372) did not exhibit solubilizing ability in MS-calcium phosphate dibasic dehydrate.

Table 2 Solubilization efficiency of different yeasts on MS-calcium phosphate dibasic dihydrate plates. Data are expressed as mean \pm SD.

\begin{tabular}{llll}
\hline Soluble phosphate level (mM) & $\mathbf{0 ~} \mathbf{~ M M}$ & $\mathbf{5 ~} \mathbf{~ M}$ & $\mathbf{2 0} \mathbf{~ m M}$ \\
\hline Meyerozyma caribbica (JYC358) & $1.6 \pm 0.31$ & $2.06 \pm 0.08$ & $1.44 \pm 0.08$ \\
Candida sp. (JYC363) & $1.72 \pm 0.2$ & $2.16 \pm 0.71$ & $2.05 \pm 0.08$ \\
Torulaspora sp. (JYC369) & $1.36 \pm 0.06$ & $1.25 \pm 0$ & $1.125 \pm 0$ \\
Cryptococcus laurentii (JYC370) & $1.19 \pm 0.07$ & $1.31 \pm 0.13$ & $1.11 \pm 0$ \\
Aureobasidium pullulans (JYC375) & $1.64 \pm 0.14$ & $2.07 \pm 0.09$ & $1.33 \pm 0$ \\
\hline
\end{tabular}

Table 3 Solubilization efficiency of different yeasts on MS-calcium phosphate tribasic plates. Data are expressed as mean \pm SD.

\begin{tabular}{llll}
\hline Soluble phosphate level $(\mathbf{m M})$ & $\mathbf{0 ~} \mathbf{~ M}$ & $\mathbf{5 ~ m M}$ & $\mathbf{2 0} \mathbf{~ m M}$ \\
\hline Meyerozyma caribbica $(\mathrm{JYC} 358)$ & $1.78 \pm 0.17$ & $1.56 \pm 0.08$ & $1.36 \pm 0.1$ \\
Candida sp. (JYC363) & $1.9 \pm 0.07$ & $1.73 \pm 0.07$ & $1.64 \pm 0.17$ \\
Torulaspora sp. (JYC369) & $1.38 \pm 0.01$ & $1.12 \pm 0.01$ & 0 \\
Cryptococcus laurentii (JYC370) & $1.34 \pm 0.01$ & $1.13 \pm 0.05$ & 0 \\
Aureobasidium pullulans (JYC375) & $1.67 \pm 0$ & $1.33 \pm 0.08$ & $1.3 \pm 0.01$ \\
\hline
\end{tabular}

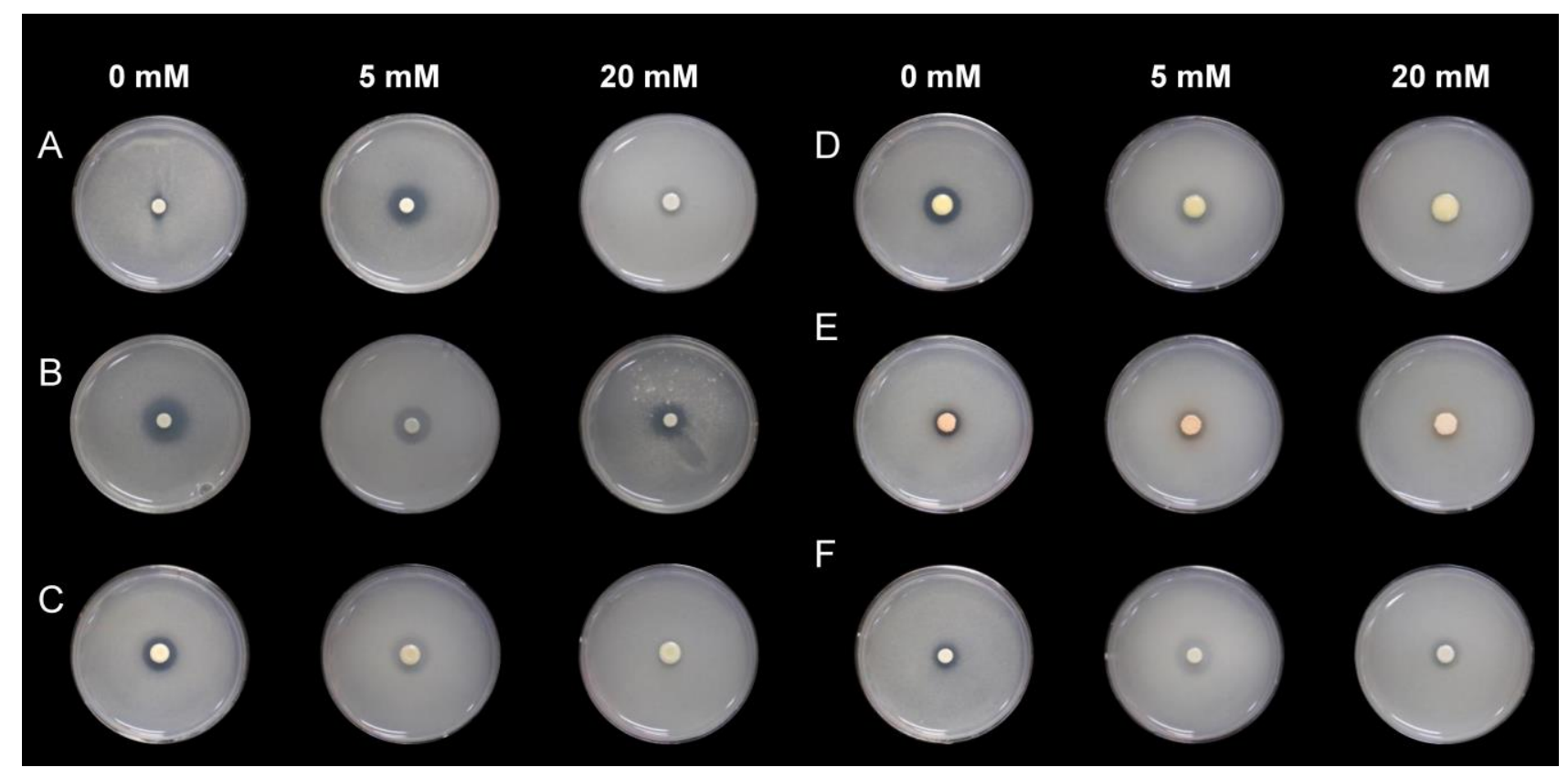

Figure 1 - Colony and phosphate solubilization characteristics of yeast strains on MS plate with calcium phosphate dibasic dihydrate. A Meyerozyma caribbica (JYC358). B Candida sp. (JYC363). C Torulaspora sp. (JYC369). D Cryptococcus laurentii (JYC370). E Pseudozyma sp. (JYC372). F Aureobasidium pullulans (JYC375). 


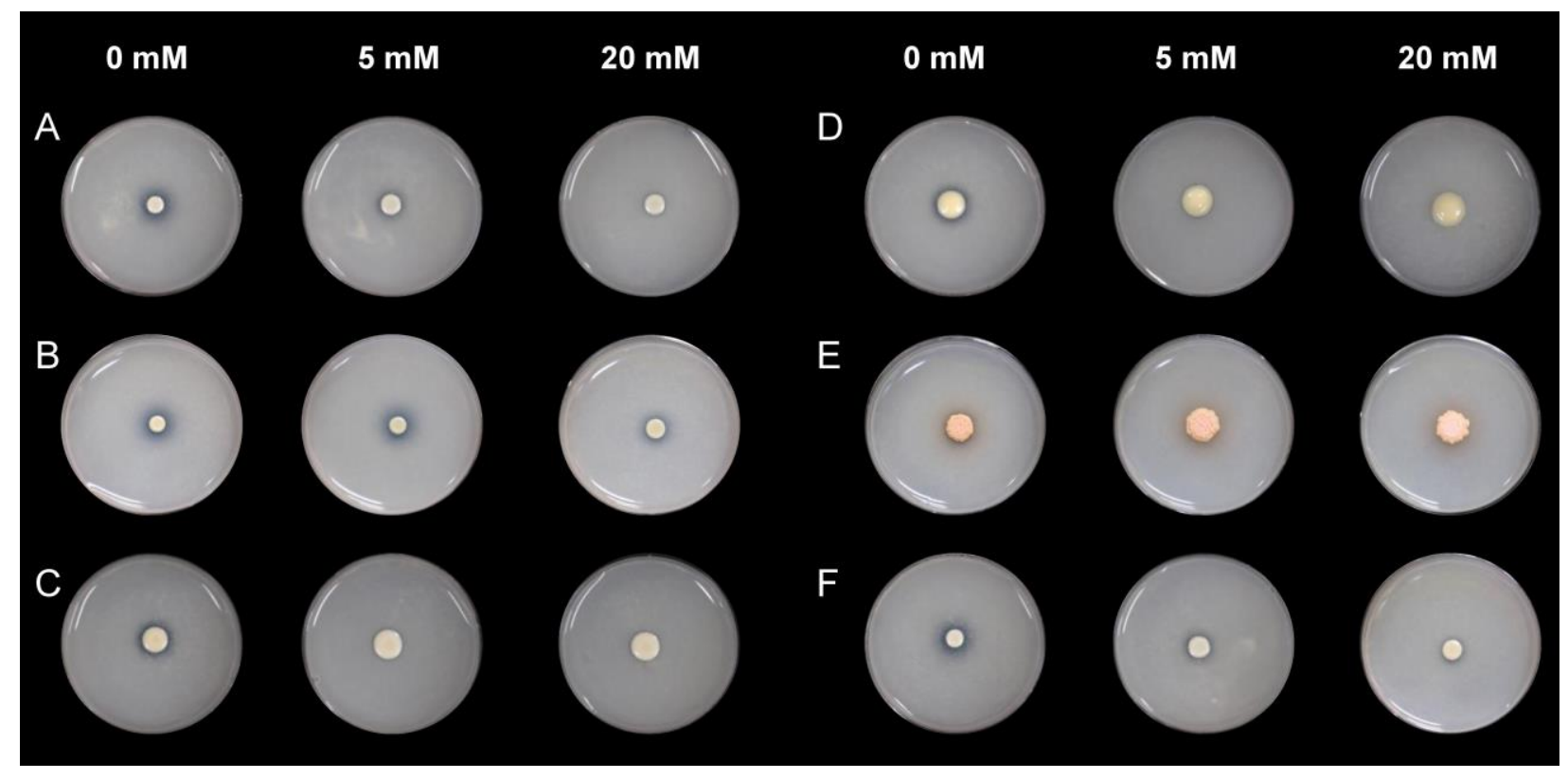

Figure 2 - Colony and phosphate solubilization characteristics of yeast strains on MS plate with calcium phosphate tribasic. A Meyerozyma caribbica (JYC358). B Candida sp. (JYC363). C Torulaspora sp. (JYC369). D Cryptococcus laurentii (JYC370). E Pseudozyma sp. (JYC372). F Aureobasidium pullulans (JYC375).

(A)

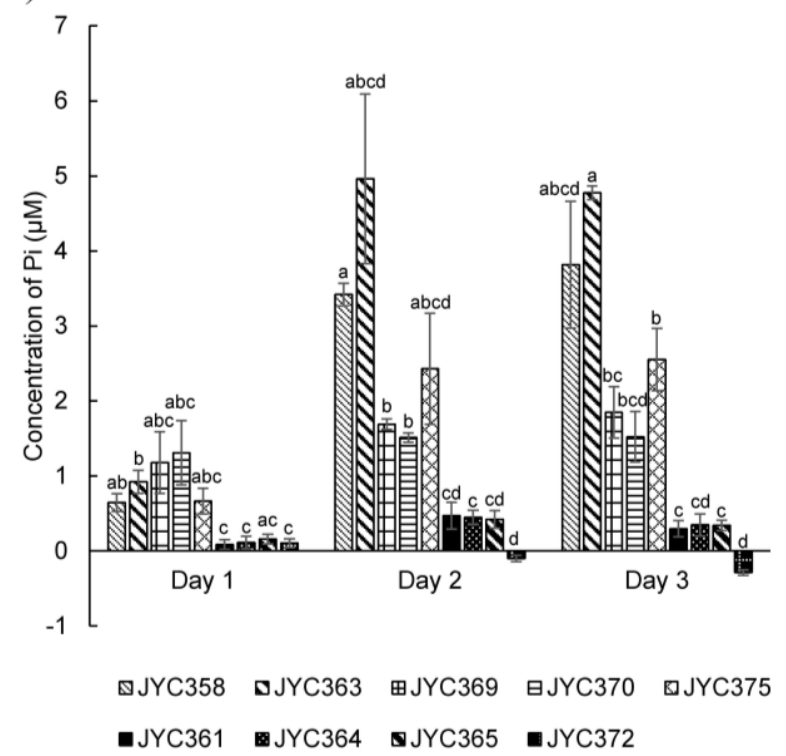

(B)

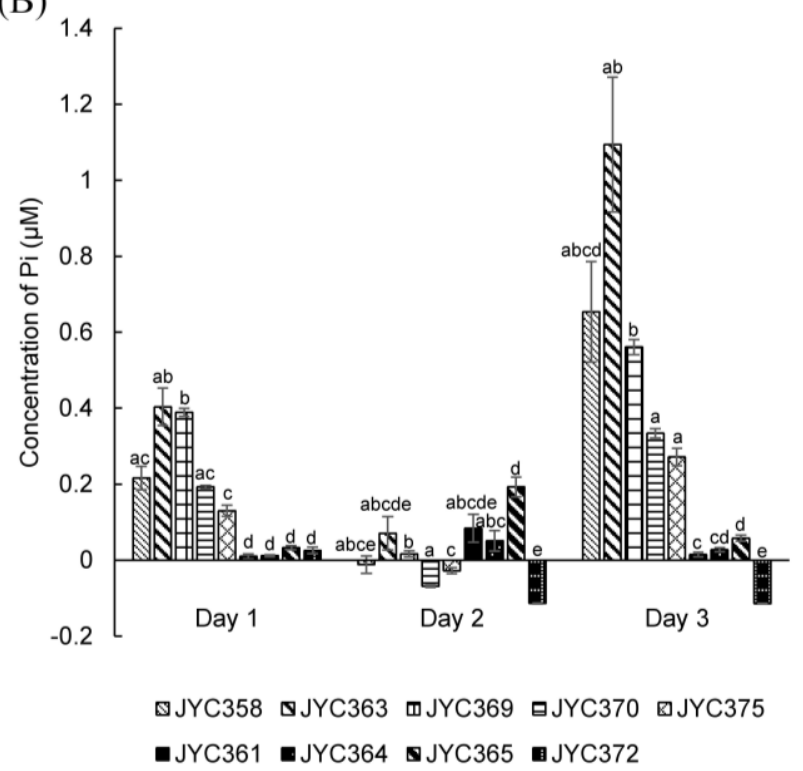

Figure 3 - Changes of phosphorus content in MS medium. A MS medium with calcium phosphate dibasic dehydrate. B MS medium with calcium phosphate tribasic. Data are the mean of three independent experiments \pm SD. Means in the same group with the same letter are not significantly different from each other in one-way ANOVA with the Games-Howell post hoc test.

In the MS-calcium phosphate tribasic liquid medium, Candida sp. (JYC363) again exhibited high solubilizing ability $(1.09 \pm 0.18 \mu \mathrm{M})$, whereas $M$. caribbica (JYC358), Torulaspora sp. (JYC369), C. laurentii (JYC370), and A. pullulans (JYC375) showed moderate solubilizing ability $(0.65 \pm 0.13,0.56 \pm 0.02,0.33 \pm 0.01$, and $0.27 \pm 0.02 \mu \mathrm{M}$, respectively). However, K. jiainicus (JYC361), Hanseniaspora uvarum (JYC364 and JYC 365), and Pseudozyma sp. (JYC372) showed almost no solubilizing ability $(0.01 \pm 0.01,0.03 \pm 0.01,0.06 \pm 0.01$, and $-0.11 \pm 0.00 \mu \mathrm{M}$, respectively). Again, the yeast growth condition of Pseudozyma sp. (JYC372) was not higher than 
that of other yeasts used in this study; therefore, Pseudozyma sp. (JYC372) did not exhibit solubilizing ability in MS-calcium phosphate tribasic medium. We also found that more phosphates were dissolved by the yeasts in MS-calcium phosphate dibasic dehydrate than in MS-calcium phosphate tribasic.

Organic acid production by yeasts led to a decrease in the $\mathrm{pH}$ of the medium. The $\mathrm{pH}$ in most treatments dropped greatly compared with the initial $\mathrm{pH}(5.97 \pm 0.01$ in calcium phosphate dibasic dihydrate, $6.12 \pm 0.02$ in calcium phosphate tribasic). The change in the $\mathrm{pH}$ of the medium was closely related to the phosphate-solubilizing ability of the yeasts. More soluble phosphate was dissolved in the medium with low pH (Fig. 4).

(A)

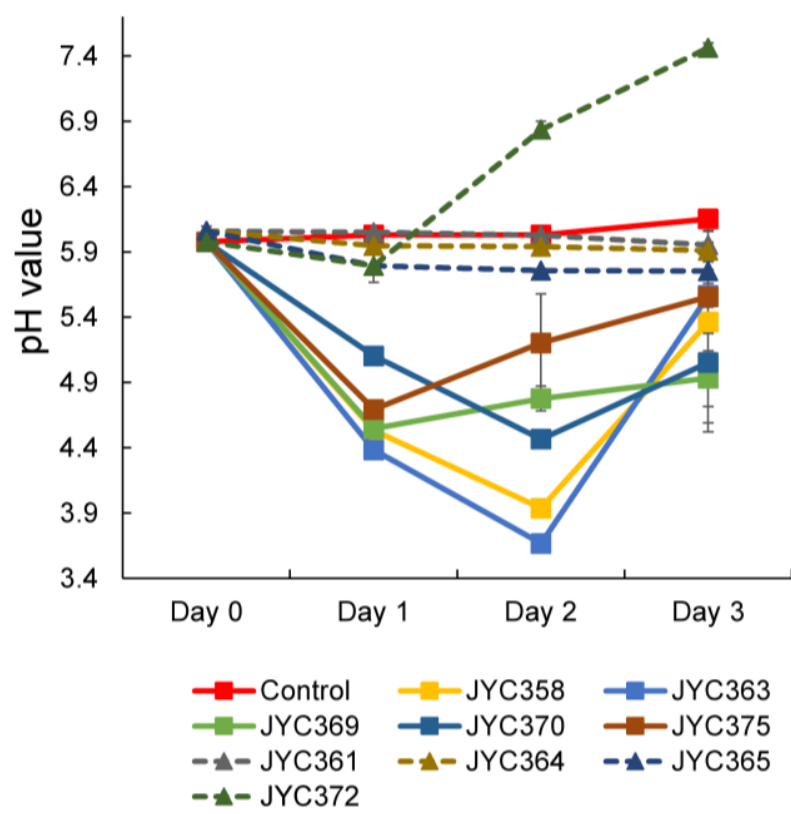

(B)

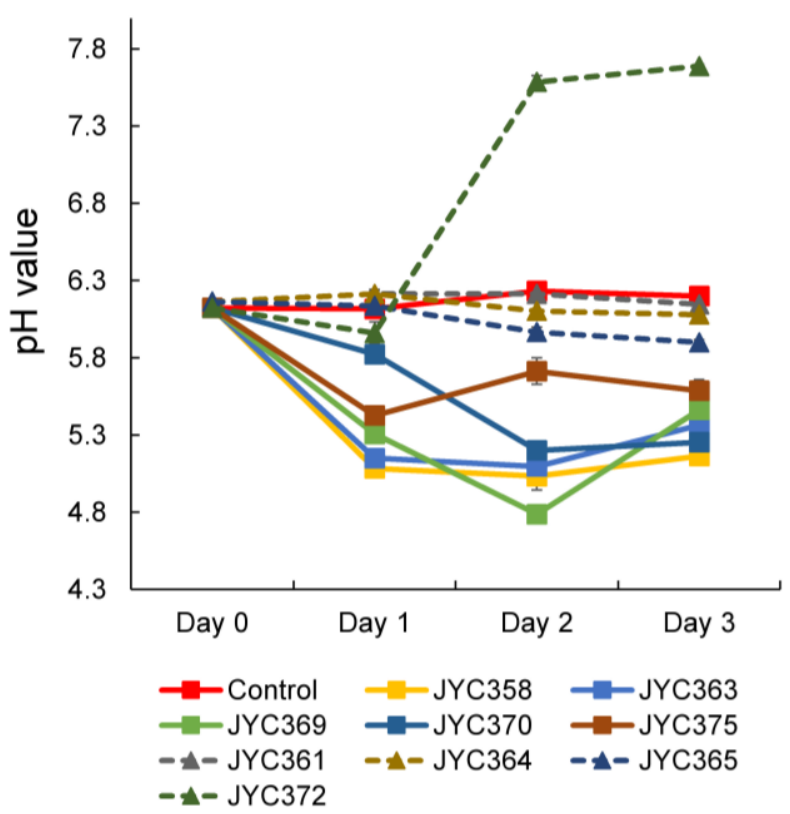

Figure 4 - Changes of $\mathrm{pH}$ in MS medium. A MS medium with calcium phosphate dibasic dehydrate. B MS medium with calcium phosphate tribasic.

\section{Effects of phosphate-solubilizing yeasts on A. thaliana growth}

To understand the effects of phosphate-solubilizing yeasts on plant growth and development, we used $A$. thaliana as a model system. Wild-type $A$. thaliana seedlings were germinated and grown on MS-calcium phosphate dibasic dehydrate plates. At 2 weeks after germination, yeast strains, including three phosphate-solubilizing strains, were inoculated $3 \mathrm{~cm}$ from the root tips, and one plate was not inoculated to serve as a negative control. M. caribbica (JYC358), Candida sp. (JYC363), and A. pullulans (JYC375) strains exhibited high phosphate-solubilizing ability, whereas Torulaspora sp. (JYC369), C. laurentii (JYC370), and Pseudozyma sp. (JYC372) strains exhibited low phosphate-solubilizing ability (Fig. 5). After 2 weeks of cocultivation of A. thaliana and $C$. laurentii (JYC370), remarkable increases in fresh weight were observed compared with controls (Fig. 6A). In particular, the cellular $\mathrm{P}_{\mathrm{i}}$ content of $A$. thaliana cocultivated with $C$. laurentii (JYC370) was much higher than that of A. thaliana cocultivated with other strains or the control group (Fig. 6B, C). Although the cellular $\mathrm{P}_{\mathrm{i}}$ content of $A$. thaliana cocultivated with Pseudozyma sp. (JYC372) or A. pullulans (JYC375) strains was higher than that of the control group, cellular $\mathrm{P}_{\mathrm{i}}$ content, fresh weight, and biomass were not significantly increased compared with those of the control group. The results suggested phosphate-solubilizing yeasts exerted beneficial effects on plant growth and development. Notably, Candida sp. (JYC363), which showed the strongest phosphate-solubilizing ability, did not show plant-development-promoting behavior in our plantyeast cocultivation assay. 


\section{Discussion}

\section{Factors influencing phosphate-solubilizing ability of yeasts}

$\mathrm{P}$ is vital for plant growth and development. Phosphate-solubilizing microorganisms play a crucial role in enhancing phosphorus availability for plants in a manner that is both environmentally friendly and sustainable (Gyaneshwar et al. 2002). The criteria used to select yeast strains for this study were their ability to solubilize calcium phosphate dibasic dihydrate and calcium phosphate tribasic on MS plates. Therefore, we selected strains that could dissolve calcium phosphate dibasic dihydrate or calcium phosphate tribasic, and some strains that could dissolve any type of calcium phosphate were used as controls. According to a previous study (Fu et al. 2016), most yeast strains can dissolve calcium phosphate dibasic dihydrate on both Pikovskaya's agar plate and MS agar plates. Surprisingly, M. caribbica (JYC358), which could not solubilize calcium phosphate dibasic dihydrate on a Pikovskaya's agar plate, dissolved calcium phosphate dibasic dihydrate on an MS agar plate. By contrast, Hanseniaspora uvarum (JYC364 and JYC 365) showed calcium phosphate tribasic -solubilizing activity on Pikovskaya's agar plate, but the ability was lost on MS- calcium phosphate tribasic plates. Therefore, the components of the medium might influence the phosphate-solubilizing ability of yeasts. Nautiyal found that some bacteria cannot solubilize tricalcium phosphate on a Pikovskaya's agar plate but can do so on a plate containing the National Botanical Research Institute's phosphate growth medium. He analyzed the components of both Pikovskaya medium and National Botanical Research Institute's phosphate growth medium to identify which component has more influence on phosphate solubilization. He found that yeast extract, which is only present in Pikovskaya medium, is the major factor reducing the phosphatesolubilizing activity of bacteria and suggested that a medium without yeast extract may be a more efficient growth medium for screening Phosphate-solubilizing microorganisms (Nautiyal 1999).

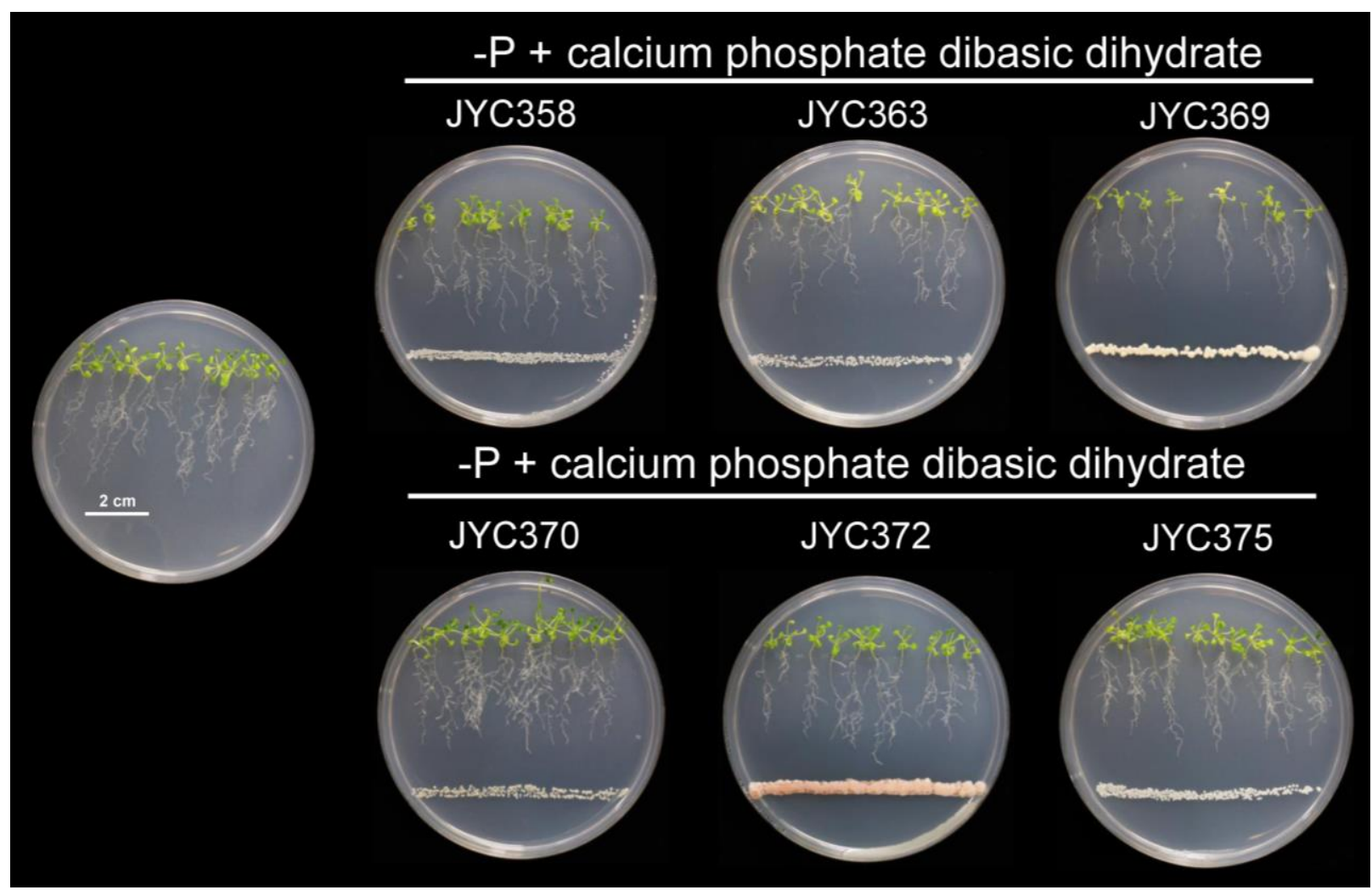

Figure 5 - Effects of phosphate-solubilizing yeasts on A. thaliana growth on $-\mathrm{P}+$ calcium phosphate dibasic dihydrate plates. Plants were inoculated with Meyerozyma caribbica (JYC358), Candida sp. (JYC363), and Torulaspora sp. (JYC369) strains exhibiting high phosphatesolubilizing ability and Cryptococcus laurentii (JYC370), Pseudozyma sp. (JYC372), and Aureobasidium pullulans (JYC375) strains exhibiting low phosphate-solubilizing ability. 
The MS medium prepared in this study also lacked yeast extract. Therefore, the absence of yeast extract in the medium may explain why the phosphate-solubilizing ability of yeasts on MScalcium phosphate dibasic dihydrate and MS-calcium phosphate tribasic differed from their phosphate-solubilizing ability on Pikovskaya's agar plate.

In this study, the phosphate-solubilizing ability of yeast strains also differed between liquid and solid media. K. jiainicus (JYC361), H. uvarum (JYC364), and H. uvarum (JYC365) solubilized phosphate in a liquid medium but did not produce a visible halo zone on MS agar plates. In previous studies, no visible halo zone on agar plates was observed for many microorganisms, but they could still solubilize insoluble inorganic phosphates in liquid media (Leyval \& Berthelin 1989, Nautiyal 1999). This can be explained by the low diffusion of the acids produced by microorganisms during growth in the solid medium (Cherif-Silini et al. 2013). These results indicate that the formation of a visible halo on agar plates is not a reliable criterion for isolating phosphate-solubilizing strains, because no visible halo zone on agar plates is detected for many isolates, but they still solubilize insoluble inorganic phosphates in liquid media.
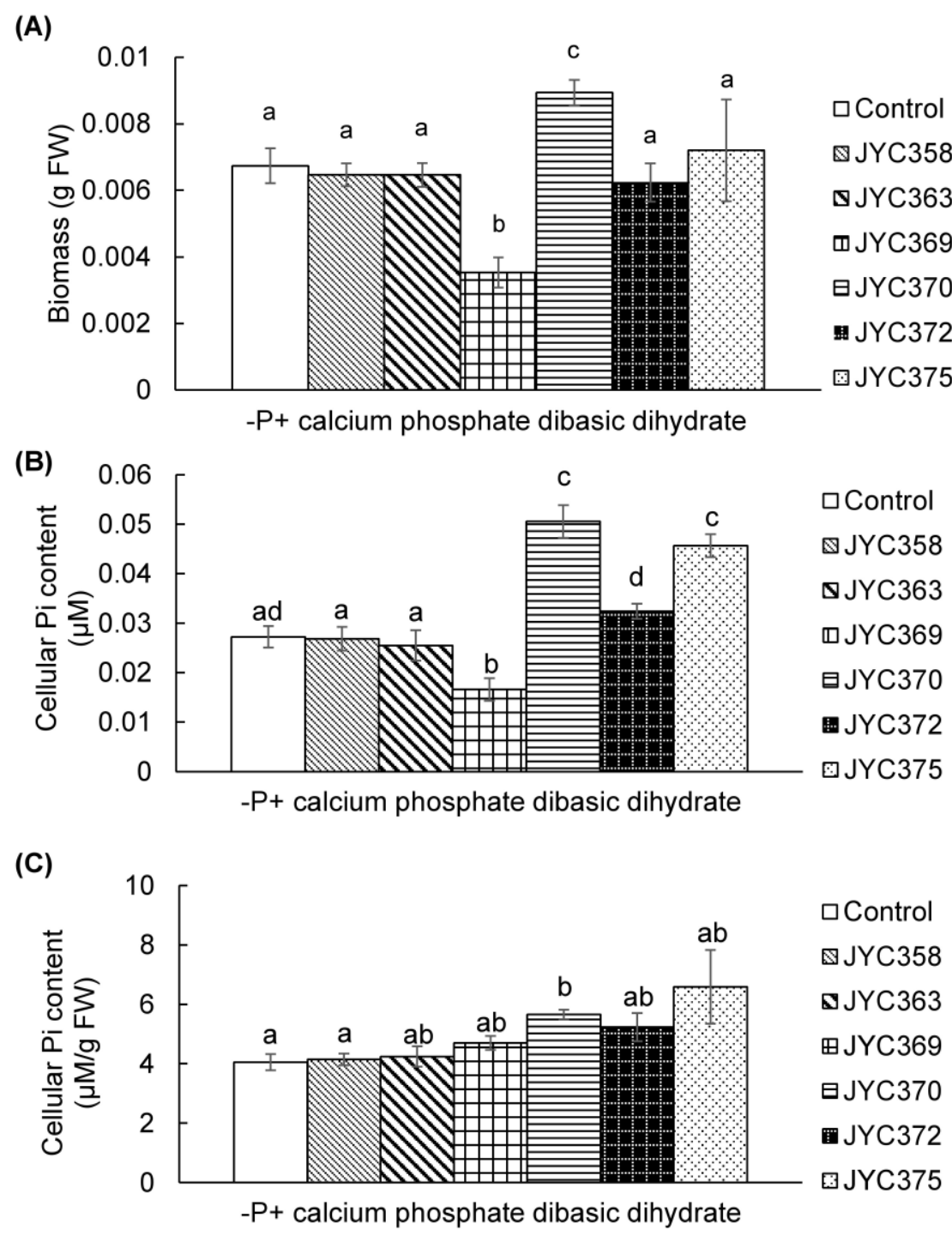

Figure 6 - Biomass and cellular $\mathrm{P}_{\mathrm{i}}$ content in A. thaliana on $-\mathrm{P}+$ calcium phosphate dibasic dihydrate plates. A Biomass. B cellular $\mathrm{P}_{\mathrm{i}}$ content in $\mu \mathrm{M}$. C cellular $\mathrm{P}_{\mathrm{i}}$ content in $\mu \mathrm{M} / \mathrm{g}$ fresh weight of $A$. thaliana in control and yeast-treated groups. Data are the mean of three independent experiments \pm SD. Means in the same group with the same letter are not significantly different from each other in one-way ANOVA with Fisher's least significant difference (A, B) or Games-Howell post hoc (C) tests. 
The $\mathrm{pH}$ of the medium inoculated with each calcium-phosphate-solubilizing yeast strain decreased. These results were probably attributed to the excretion of $\mathrm{H}^{+}$or the production of organic acids by the yeasts. A previous study found that Arthrobacter sp. CC-BC03, which dissolved the most phosphorus, acidified the $\mathrm{pH}$ of the medium the most (Chen et al. 2006), similar to our result. In addition, many studies have shown that organic acids can solubilize more phosphate than can inorganic acids at the same $\mathrm{pH}$ possibly because of chelation. Illmer and Schinner found that artificial acidification of culture media with $\mathrm{HCl}$ solubilized less calcium phosphate than was solubilized by either Penicillium sp. or Pseudomonas sp. (Illmer \& Schinner 1992). However, the detailed mechanisms of yeast phosphate solubilization found in the present study require further investigation through high-performance liquid chromatography to analyze which organic acids are produced by the candidate strains.

\section{Phosphate solubilization at different levels of soluble phosphate}

The growth rate of microorganisms is related to the level of environmental soluble phosphate (Thomas et al. 2011). Zeng et al. found that the phosphate-solubilizing ability of Burkholderia multivorans WS-FJ9 bacteria gradually decreased with a further increase in soluble phosphate levels, and the phosphate-solubilizing activity of the strain was completely inhibited in the presence of $20 \mathrm{mM}$ exogenous soluble phosphate (Zeng et al. 2017). Through transcriptome profiling of $B$. multivorans WS-FJ9 grown in the presence of three levels of exogenous soluble phosphate, they determined that some genes related to cell growth were continuously upregulated, which would account for the improved growth of WS-FJ9 in the presence of high levels of soluble phosphate. Interestingly, they found that the genes related to glucose metabolism, including glycerate kinase, 2-oxoglutarate dehydrogenase, and sugar ABC-type transporter, were continuously downregulated, indicating that metabolic channeling of glucose toward the phosphorylative pathway is negatively regulated by soluble phosphate. In this study, the phosphate-solubilizing activity of some yeasts was also completely inhibited in the presence of high concentrations of exogenous soluble phosphate, suggesting that some yeasts can sense the concentration of exogenous soluble phosphate and can regulate their phosphate-solubilizing ability through metabolic rewiring. Thus, these strains are superior candidates for biofertilizer because they do not expend energy to produce organic acids and may instead expend energy to increase their population in soils.

\section{Enhanced growth of plants by the yeasts}

Studies have shown that plants inoculated with phosphate-solubilizing bacteria demonstrate higher development than un-inoculated plants under soluble phosphate limiting conditions (Gurdeep \& Reddy 2015, Otieno et al. 2015). Our study similarly showed that $C$. laurentii (JYC370) enhanced the growth and cellular $\mathrm{P}_{\mathrm{i}}$ content of plants on MS-calcium phosphate dibasic dihydrate plates. This finding suggests that plants can absorb soluble phosphate dissolved by $C$. laurentii (JYC370) and can convert it into biomass. We also found C. laurentii (JYC370) exhibited calcium phosphate tribasic-solubilizing activity. Most importantly, the phosphate-solubilizing activity of $C$. laurentii (JYC370) was flexible and was negatively regulated by soluble phosphate. In our previous study, C. laurentii (JYC370) showed other plant-growth-promoting characteristics, such as production of indole-3-acetic acid, solubilization of zinc oxide, production of $\mathrm{NH}_{3}$, catalase activity, and chitinase activity (Fu et al. 2016). C. laurentii is often associated with plants and is common in soil samples from fields (Sláviková \& Vadkertiová 2003). C. laurentii can grow oligotrophically and is adequately adapted for survival in soil (Kimura et al. 1998). Thus, $C$. laurentii has been suggested to be competitive soil yeasts and a candidate biofertilizer. Cloete et al. (2009) reported that $C$. laurentii increased root growth by $51 \%$ when inoculated into seedlings of Agathosma betulina, a medicinal plant adapted to low-nutrient soils. C. laurentii has also been reported to have great commercial potential as a biological control agent against the postharvest diseases blue mold and brown rot, which are caused by Penicillium expansum and Monilinia fructicola, respectively, in sweet cherry fruit (Qin \& Tian 2005) and postharvest diseases in other fruits, such as strawberries, kiwifruit, and table grapes (Lima et al. 1998). Benbow \& Sugar (1999) 
identified the potential of $C$. laurentii for biocontrol of postharvest diseases in pears through preharvest application.

\section{Correlation between phosphate-solubilizing ability and plant growth promotion}

Phosphate-solubilizing microorganisms can promote plant growth and increase crop yield. Comparisons of the characteristics of phosphate-solubilizing microorganisms isolated from one ecological niche have shown their differences in phosphate-solubilizing ability (Taurian et al. 2010, Zhang et al. 2017), raising the question of whether higher phosphate-solubilizing ability is correlated with higher plant growth promotion. The growth of Pisum sativum was promoted by phosphate-solubilizing endophytic Pseudomonas isolates (Otieno et al. 2015) capable of solubilizing inorganic phosphate $\left(\mathrm{Ca}_{3}\left(\mathrm{PO}_{4}\right)_{2}\right)$. Among these isolates, the L132 strain exhibited higher phosphate-solubilizing ability than L321. However, the biomass of $P$. sativum plants inoculated with the strain L321 was higher than that of plants inoculated L132 (Otieno et al. 2015). Additionally, five of the Pseudomonas rhizobacteria strains naturally associated with A. thaliana were isolated, and their phosphate-solubilizing ability was characterized (Schwachtje et al. 2012). The Pseudomonas G53 strain exhibited higher phosphate-solubilizing ability than G62. The G53 and G62 strains had contrasting effects on plant growth; the growth of Arabidopsis plants was promoted by G62 and inhibited by G53 (Schwachtje et al. 2012). In this study, the yeast strain JYC363 displayed higher phosphate-solubilizing ability, whereas JYC370 showed moderate solubilizing ability (Fig. 3). The yeast strain JYC370 enhanced the growth of $A$. thaliana more than JYC363 did, indicating that this phosphate-solubilizing property is a characteristic promoting plant growth (Fig. 5). These results suggest that the higher phosphate-solubilizing ability of phosphatesolubilizing microorganisms may not be attributed to plant growth promotion. Integration of multiple microbial strains as a single consortium can offer different benefits to crop plants.

In summary, some yeast strains can regulate phosphate solubilization according to exogenous soluble phosphate. Among the yeast strains in this study, C. laurentii (JYC370) significantly enhanced the growth of $A$. thaliana. These results revealed the potential of $C$. laurentii (JYC370) for biofertilizer applications and commercial use in the field. However, field trials should be conducted, and the mechanisms underlying the calcium-phosphate-solubilizing activity of $C$. laurentii (JYC370) require additional study to verify the practical and effective use of $C$. laurentii as biofertilizer.

\section{Acknowledgments}

We thank the members of the Chou Lab for their contribution to the manuscript. This work was supported by grants from the Ministry of Science and Technology (MOST 105-2311-B-018001-MY3 to J.-Y. Chou; MOST 104-2311-B-018-003-MY3 to S.-F. Fu). This manuscript was edited by Wallace Academic Editing.

\section{References}

Abhilash P, Dubey RK, Tripathi V, Gupta VK, Singh HB. 2016 - Plant growth-promoting microorganisms for environmental sustainability. Trends in Biotechnology 34, 847-850.

Ames BN. 1966 - Assay of inorganic phosphate, total phosphate and phosphatases. Methods in Enzymology 8, 115-118.

Amprayn Ko, Rose MT, Kecskés M, Pereg L et al. 2012 - Plant growth promoting characteristics of soil yeast (Candida tropicalis $\mathrm{HY}$ ) and its effectiveness for promoting rice growth. Applied Soil Ecology 61, 295-299.

Behera B, Yadav H, Singh S, Mishra R et al. 2017 - Phosphate solubilization and acid phosphatase activity of Serratia sp. isolated from mangrove soil of Mahanadi river delta, Odisha, India. Journal of Genetic Engineering and Biotechnology 15, 169-178.

Belgaroui N, Berthomieu P, Rouached H, Hanin M. 2016 - The secretion of the bacterial phytase PHY-US 417 by Arabidopsis roots reveals its potential for increasing phosphate acquisition 
and biomass production during co-growth. Plant Biotechnology Journal 14, 1914-1924.

Benbow JM, Sugar D. 1999 - Fruit surface colonization and biological control of postharvest diseases of pear by preharvest yeast applications. Plant Disease 83, 839-844.

Chen Y, Rekha P, Arun A, Shen F et al. 2006 - Phosphate solubilizing bacteria from subtropical soil and their tricalcium phosphate solubilizing abilities. Applied Soil Ecology 34, 33-41.

Cherif-Silini H, Silini A, Ghoul M, Yahiaoui B, Arif F. 2013 - Solubilization of phosphate by the Bacillus under salt stress and in the presence of osmoprotectant compounds. African Journal of Microbiology Research 7, 4562-4571.

Cloete KJ, Valentine AJ, Stander MA, Blomerus LM, Botha A. 2009 - Evidence of symbiosis between the soil yeast Cryptococcus laurentii and a sclerophyllous medicinal shrub, Agathosma betulina (Berg.) Pillans. Microbial Ecology 57, 624-632.

Cruz-Ramírez A, Oropeza-Aburto A, Razo-Hernández F, Ramírez-Chávez E, Herrera-Estrella L. 2006 - Phospholipase DZ2 plays an important role in extraplastidic galactolipid biosynthesis and phosphate recycling in Arabidopsis roots. Proceedings of the National Academy of Sciences 103, 6765-6770.

Fu SF, Sun PF, Lu HY, Wei JY et al. 2016 - Plant growth-promoting traits of yeasts isolated from the phyllosphere and rhizosphere of Drosera spatulata Lab. Fungal Biology 120, 433-448.

Gaind S, Nain L. 2015 - Soil-phosphorus mobilization potential of phytate mineralizing fungi. Journal of plant nutrition 38, 2159-2175.

Gilbert N. 2009 - Environment: the disappearing nutrient. Nature News 461, 716-718.

Goldstein AH. 1995 - Recent progress in understanding the molecular genetics and biochemistry of calcium phosphate solubilization by gram negative bacteria. Biological Agriculture \& Horticulture 12, 185-193.

Gurdeep K, Reddy MS. 2015 - Effects of phosphate-solubilizing bacteria, rock phosphate and chemical fertilizers on maize-wheat cropping cycle and economics. Pedosphere 25, 428-437.

Gyaneshwar P, Kumar GN, Parekh L, Poole P. 2002 - Role of soil microorganisms in improving P nutrition of plants. Plant and Soil 245, 83-93.

Hameeda B, Harini G, Rupela O, Wani S, Reddy G. 2008 - Growth promotion of maize by phosphate-solubilizing bacteria isolated from composts and macrofauna. Microbiological Research 163, 234-242.

Hermans C, Hammond JP, White PJ, Verbruggen N. 2006 - How do plants respond to nutrient shortage by biomass allocation? Trends in Plant Science 11, 610-617.

Hinsinger P. 2001 - Bioavailability of soil inorganic P in the rhizosphere as affected by rootinduced chemical changes: a review. Plant and Soil 237, 173-195.

Hu XJ, Li ZJ, Cao YC, Zhang J et al. 2010 - Isolation and identification of a phosphate-solubilizing bacterium Pantoea stewartii subsp. stewartii g6, and effects of temperature, salinity, and $\mathrm{pH}$ on its growth under indoor culture conditions. Aquaculture International 18, 1079-1091.

Illmer P, Schinner F. 1992 - Solubilization of inorganic phosphates by microorganisms isolated from forest soils. Soil Biology and Biochemistry 24, 389-395.

Kimura Y, Nakano Y, Fujita K, Miyabe S et al. 1998 - Isolation and characteristics of yeasts able to grow at low concentrations of nutrients. Yeast 14, 233-238.

Kumar VV. 2016 - Plant growth-promoting microorganisms: interaction with plants and soil. Pages 1-16 Plant, Soil and Microbes. Springer.

Leyval C, Berthelin J. 1989 - Interactions between Laccaria laccata, Agrobacterium radiobacter and beech roots: Influence on $\mathrm{P}, \mathrm{K}, \mathrm{Mg}$, and Fe mobilization from minerals and plant growth. Plant and Soil 117, 103-110.

Lima G, Curtis F de, Castoria R., Cicco V de. 1998 - Activity of the yeasts Cryptococcus laurentii and Rhodotorula glutinis against post-harvest rots on different fruits. Biocontrol Science and Technology 8, 257-267.

Mardad I, Serrano A, Soukri A. 2013 - Solubilization of inorganic phosphate and production of organic acids by bacteria isolated from a Moroccan mineral phosphate deposit. African Journal of Microbiology Research 7, 626-635. 
Meena RS, Meena PD, Yadav GS, Yadav SS. 2017 - Phosphate solubilizing microorganisms, principles and application of microphos technology. Journal of Cleaner Production 145, 157 158.

Nalini P, Ellaiah P, Prabhakar T, Girijasankar G. 2015 - Microbial alkaline phosphatases in bioprocessing. International Journal of Current Microbiology and Applied Sciences 4, 384396.

Nautiyal CS. 1999 - An efficient microbiological growth medium for screening phosphate solubilizing microorganisms. FEMS Microbiology Letters 170, 265-270.

Nussaume L, Kanno S, Javot H, Marin E et al. 2011 - Phosphate import in plants: focus on the PHT1 transporters. Frontiers in Plant Science 2, 83.

Otieno N, Lally RD, Kiwanuka S, Lloyd A, Ryan D, Germaine KJ, Dowling DN. 2015 - Plant growth promotion induced by phosphate solubilizing endophytic Pseudomonas isolates. Frontiers in Microbiology 6, 745 .

Patel DK, Archana G, Kumar GN. 2008 - Variation in the nature of organic acid secretion and mineral phosphate solubilization by Citrobacter sp. DHRSS in the presence of different sugars. Current Microbiology 56, 168-174.

Ponmurugan P, Gopi C. 2006 - In vitro production of growth regulators and phosphatase activity by phosphate solubilizing bacteria. African Journal of Biotechnology 5, 348-350.

Qin GZ, Tian SP. 2005 - Enhancement of biocontrol activity of Cryptococcus laurentii by silicon and the possible mechanisms involved. Phytopathology 95, 69-75.

Raghothama K. 1999 - Phosphate acquisition. Annual Review of Plant Biology 50, 665-693.

Rodríguez H, Fraga R. 1999 - Phosphate solubilizing bacteria and their role in plant growth promotion. Biotechnology Advances 17, 319-339.

Saxena J, Chandra S, Nain L. 2013 - Synergistic effect of phosphate solubilizing rhizobacteria and arbuscular mycorrhiza on growth and yield of wheat plants. Journal of Soil Science and Plant Nutrition 13, 511-525.

Schwachtje J, Karojet S, Kunz S, Brouwer S, van Dongen JT. 2012 - Plant-growth promoting effect of newly isolated rhizobacteria varies between two Arabidopsis ecotypes. Plant Signaling \& Behavior 7, 623-627.

Sláviková E, Vadkertiová R. 2003 - The diversity of yeasts in the agricultural soil. Journal of Basic Microbiology 43, 430-436.

Sultenfuss J, Doyle W. 1999 - Functions of phosphorus in plants. Better Crops 83, 6-7.

Taurian T, Anzuay MS, Angelini JG, Tonelli ML et al. 2010 - Phosphate-solubilizing peanut associated bacteria: screening for plant growth-promoting activities. Plant and Soil 329, 421431.

Thomas L, Hodgson DA, Wentzel A, Nieselt K et al. 2011 - Metabolic switches and adaptations deduced from the proteomes of Streptomyces coelicolor wild type and phoP mutant grown in batch culture. Molecular \& Cellular Proteomics M111, 013797.

Vyas P, Gulati A. 2009 - Organic acid production in vitro and plant growth promotion in maize under controlled environment by phosphate-solubilizing fluorescent Pseudomonas. BMC Microbiology 9, 174.

Wang L, Dong J, Gao Z, Liu D. 2012 - The Arabidopsis gene hypersensitive to phosphate starvation 3 encodes ethylene overproduction 1. Plant and Cell Physiology 53, 1093-1105.

Whitelaw MA. 1999 - Growth promotion of plants inoculated with phosphate-solubilizing fungi. Advances in Agronomy 69, 99-151.

Wrage N, Chapuis-Lardy L, Isselstein J. 2010 - Phosphorus, plant biodiversity and climate change. Pages 147-169 Sociology, Organic Farming, Climate Change and Soil Science. Springer.

Yu X, Liu X, Zhu TH, Liu GH, Mao C. 2012 - Co-inoculation with phosphate-solubilzing and nitrogen-fixing bacteria on solubilization of rock phosphate and their effect on growth promotion and nutrient uptake by walnut. European Journal of Soil Biology 50, 112-117.

Zeng Q, Wu X, Wang J, Ding X. 2017 - Phosphate solubilization and gene expression of phosphate-solubilizing bacterium Burkholderia multivorans WS-FJ9 under different levels of 
soluble phosphate. J. Microbiol. Biotechnol 27, 844-855.

Zhang J, Wang P, Fang L, Zhang QA et al. 2017 - Isolation and characterization of phosphatesolubilizing bacteria from mushroom residues and their effect on tomato plant growth promotion. Polish Journal of Microbiology 66, 57-65. 